\title{
106. DEUTSCHER BIBLIOTHEKARTAG
}

(FRANKFURT AM MAIN, 30. MAI-2. JUNI 2017)

Ort: Congress Center, Frankfurt am Main, Deutschland Website: http://bibliothekartag2017.de/

\section{$38^{\text {TH }}$ ANNUAL IATUL CONFERENCE (BOZEN, 18.-22. JUNI 2017)}

Leitthema: „Embedding Libraries - Service and Development in Context“ Ort: Freie Universität Bozen, Südtirol, Italien

Website: https://iatulevents.ub.tum.de/ocs/index.php/bozen2017/ bozen 2017/schedConf/registration

\section{6 $^{\text {TH }}$ ANNUAL LIBER CONFERENCE (PATRAS, 5.-7. JULI 2017)}

Leitthema: „Libraries Powering Sustainable Knowledge in the Digital Age“ Ort: Conference and Cultural Centre, Patras, Griechenland Website: http://liber2017.lis.upatras.gr/

\section{3. ÖSTERREICHISCHER BIBLIOTHEKARTAG (LINZ, 12.-15. SEPTEMBER 2017)}

Leitthema: „Wolkenkuckucksheim. Bibliotheken in der Cloud“ Ort: Johannes Kepler Universität, Linz, Österreich Website: http://bibliothekartag2017.univie.ac.at/home/

\section{EUROPEAN CONFERENCE ON INFORMATION LITERACY (ECIL)} (SAINT-MALO, 18.-21. SEPTEMBER 2017)

Leitthema: „Workplace Information Literacy“

Ort: Saint-Malo, Frankreich

Website: http://ecil2017.ilconf.org/

\section{THE FUTURE OF RESEARCH COMMUNICATIONS AND E-SCHO- LARSHIP (FORCE) (BERLIN, 25.-27. OKTOBER 2017)}

Ort: Kalkscheune, Berlin, Deutschland Website: https://www.force11.org/meetings/force2017 